\title{
Theoretical and Experimental Insights into the Possible Interfacial Interactions between $\beta$-Glucan and Fat Molecules in Aqueous Media
}

\author{
Tamanna Islama,c, Md. Nurul Huda, ${ }^{a, c}$, Md Ariful Ahsan ${ }^{\mathrm{b}}$, Humayra Afrin ${ }^{\mathrm{a}, \mathrm{c}}$, Christiancel \\ Joseph J Salazara, Md Nurunnabi ${ }^{a, c, d}$ \\ a Environmental Science \& Engineering Program, University of Texas at El Paso, TX 79968, \\ United States
}

${ }^{b}$ Department of Chemistry and Biochemistry, College of Sciences, University of Texas at El Paso, TX 79968, United States

${ }^{c}$ Department of Pharmaceutical Sciences, School of Pharmacy, University of Texas at El Paso, TX 79902, United States

${ }^{\mathrm{d}}$ Border Biomedical Research Center, University of Texas at El Paso, TX 79968, United States

*Address for correspondence:

Dr. Md Nurunnabi

Department of Pharmaceutical Sciences

University of Texas at El Paso School of Pharmacy

1101 N. Campbell St. El Paso, Texas 79902, USA

Email: $\underline{\text { mnurunnabi@utep.edu }}$

Phone: 915-747-8335 
(A)

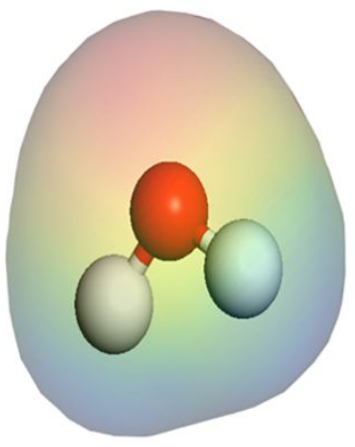

(B)

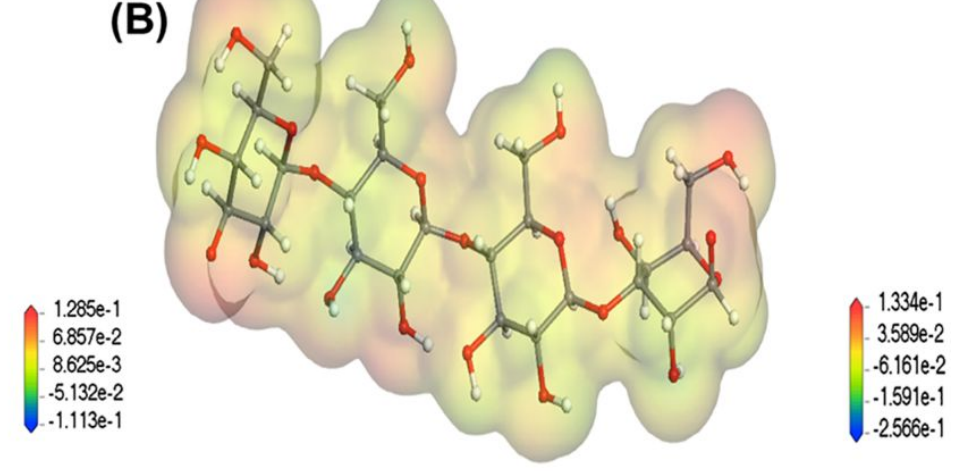

(C)

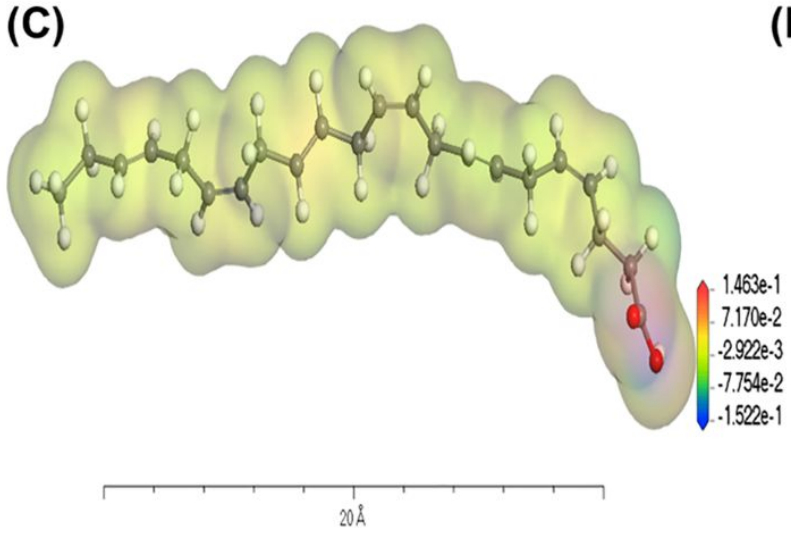

(D)
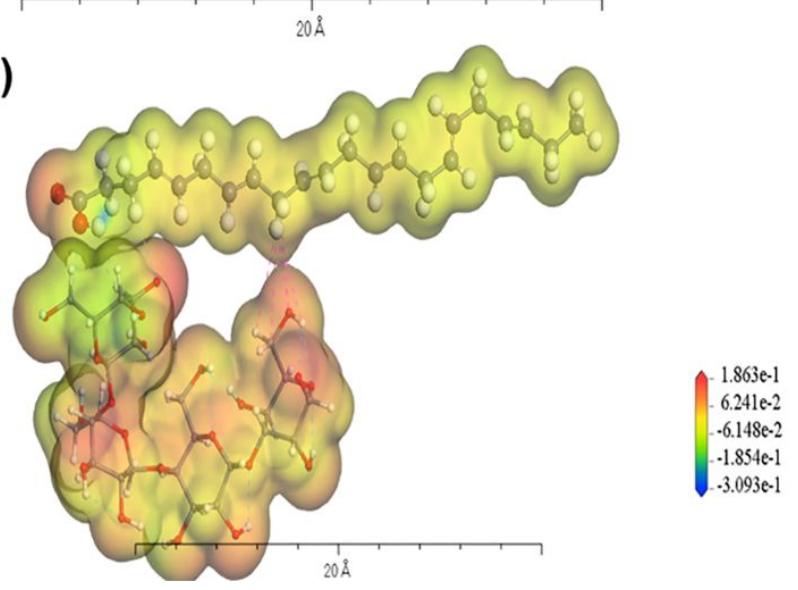

Supporting Figure S1. Electron density distribution profile. The electron density profiles for (A) $\mathrm{H}_{2} \mathrm{O}$, (B) BG, (C) DHA, and (D) BG-DHA system are shown. The color bars at the right-hand side show the polarity profile for the systems. 
(A)

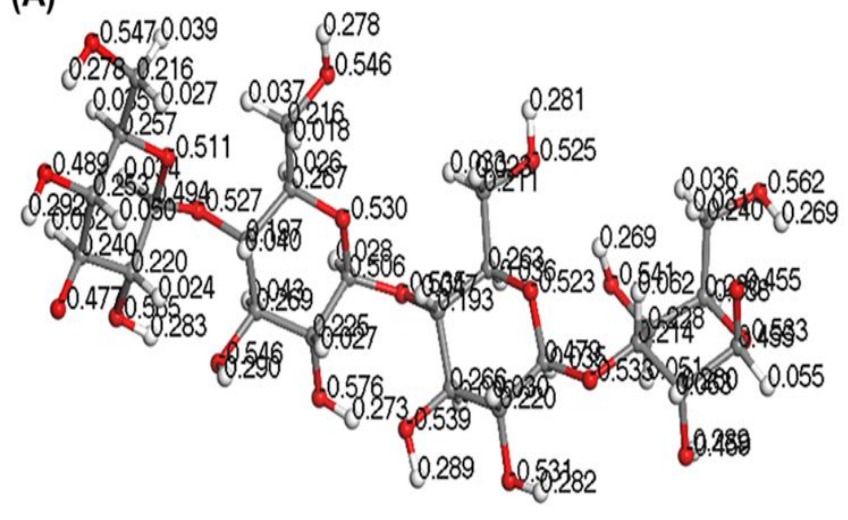

(B)

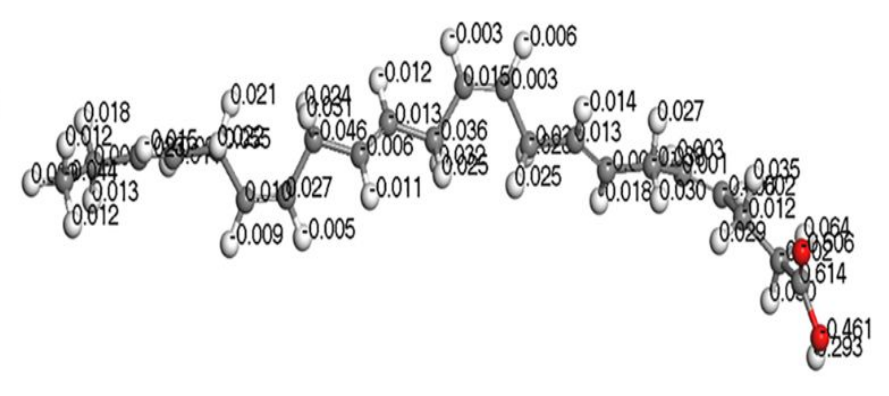

(C)
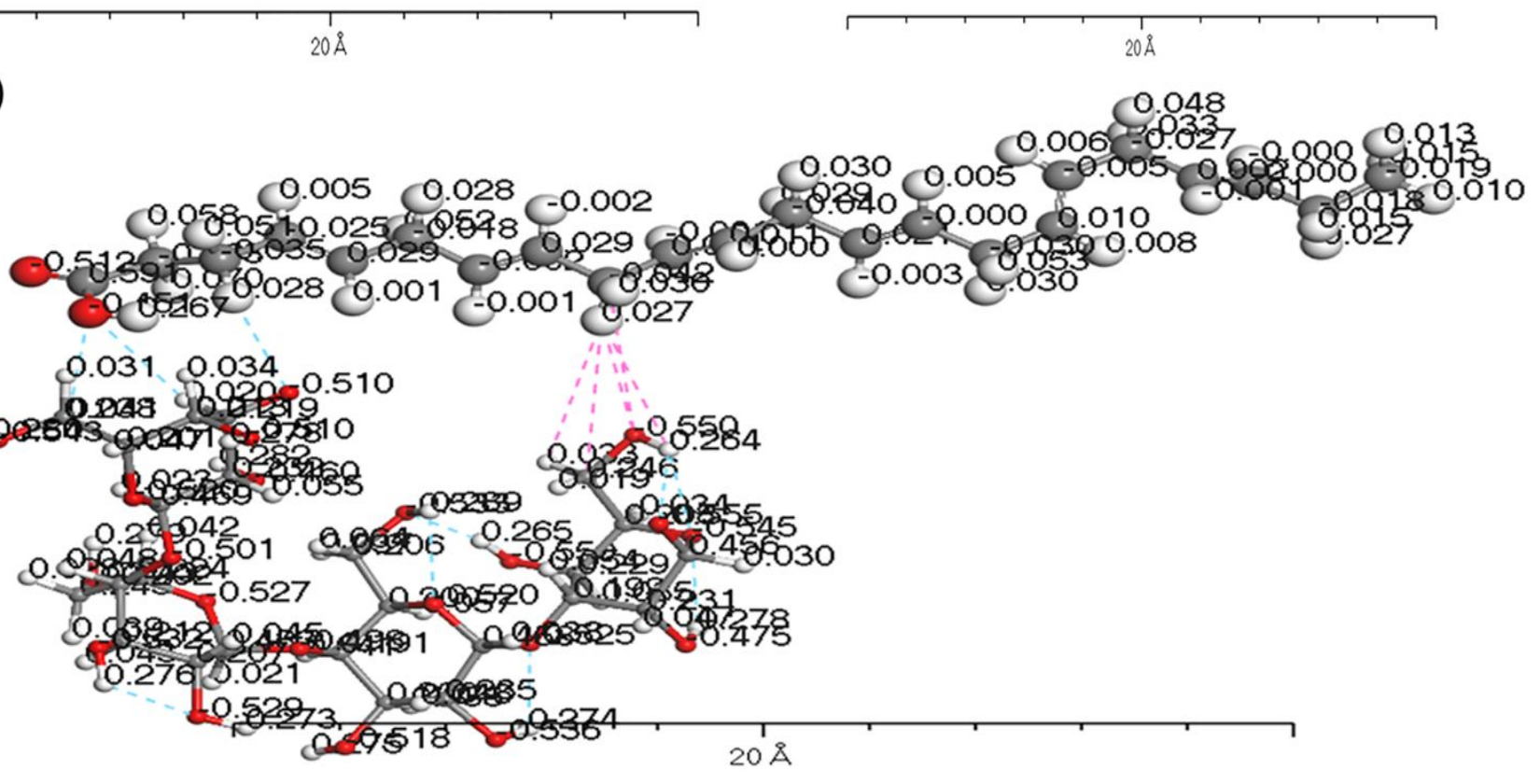

Supporting Figure S2. Mulliken charge distribution profile. The atomic charges for (A) BG, (B) DHA, and (C) BG-DHA system are shown. 


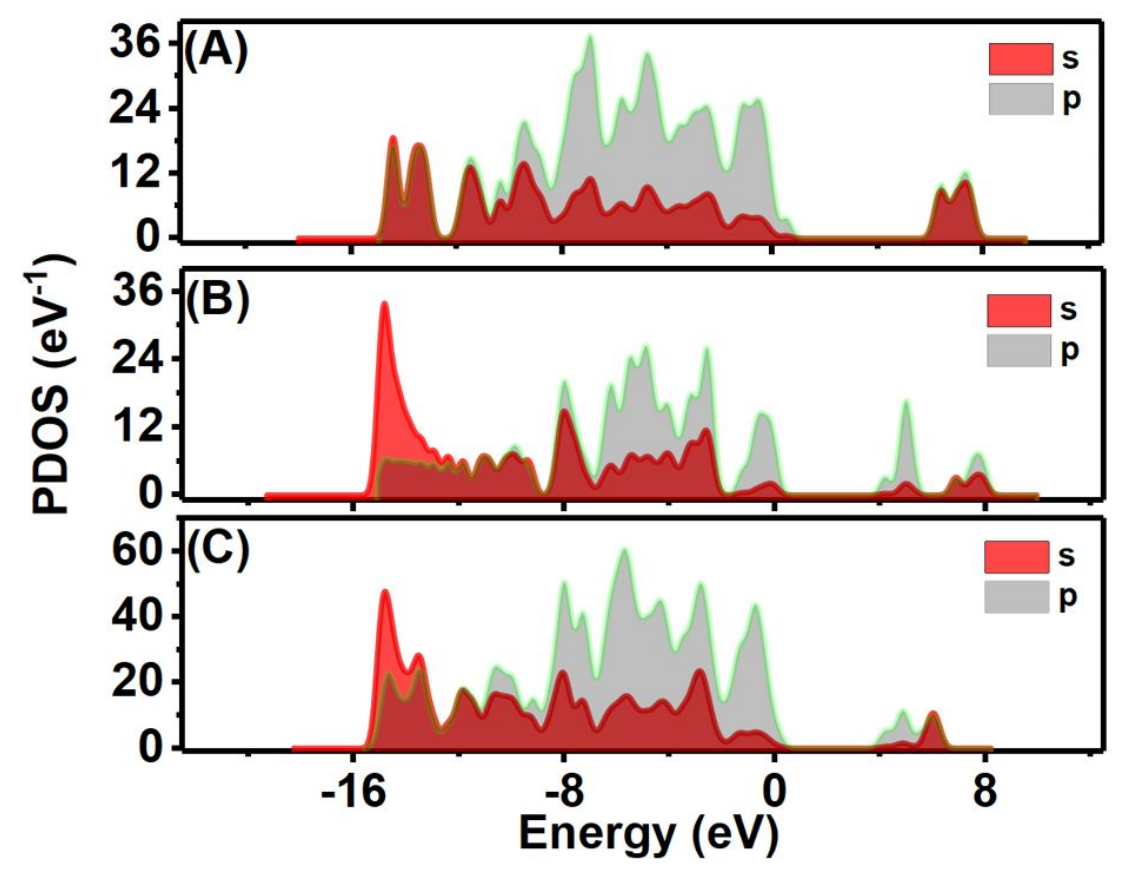

Supporting Figure S3. The PDOS plots. (A), (B), and (C) show the PDOS for BG, DHA, and BG-DHA.

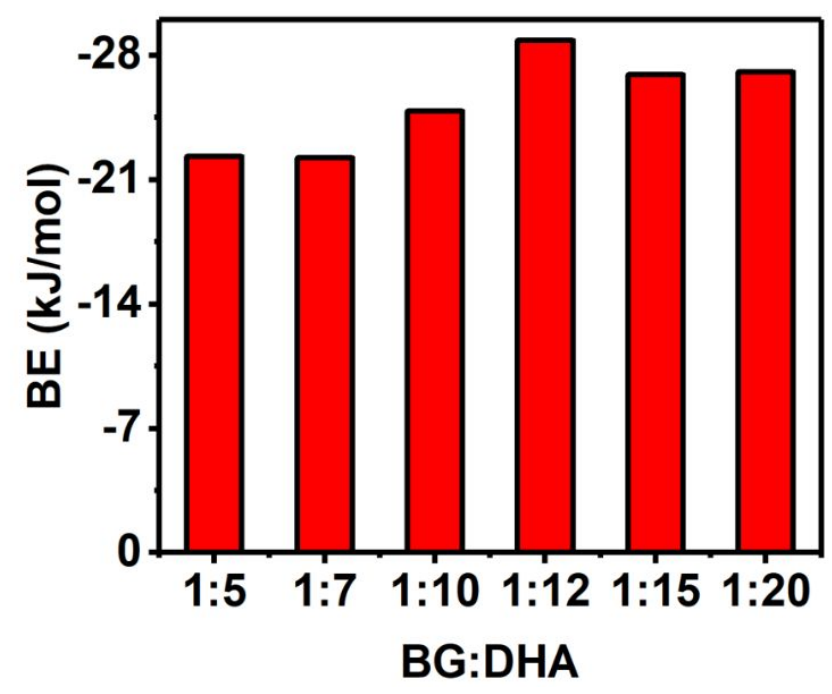

Supporting Figure S4. Binding energy obtained for six systems containing both DHA and $\mathrm{H}_{2} \mathrm{O}$ through adsorption locator simulation. 


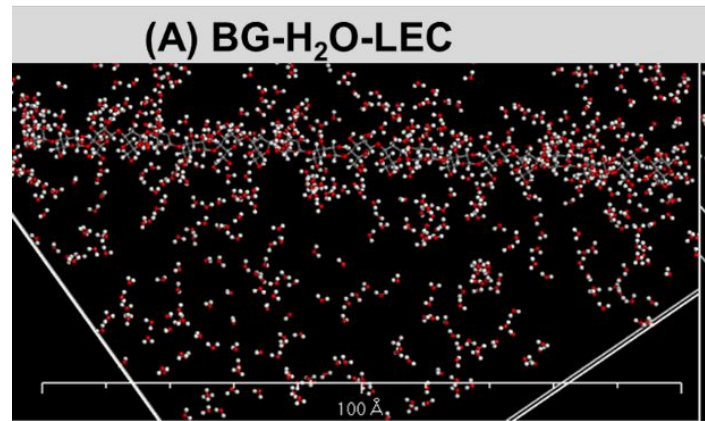

(C) BG-DHA-LEC

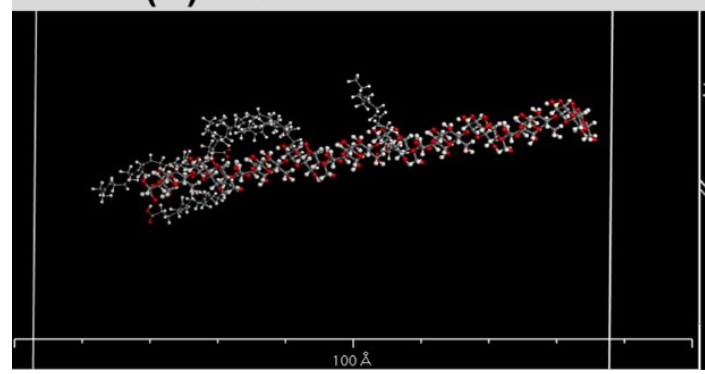

(B) $\mathrm{BG}-\mathrm{H}_{2} \mathrm{O}-\mathrm{HEC}$

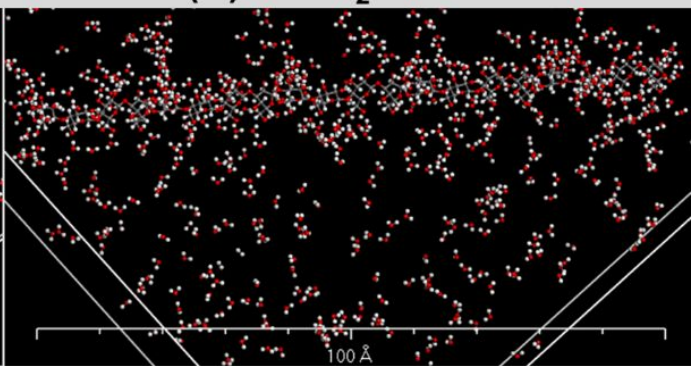

(D) BG-DHA-HEC

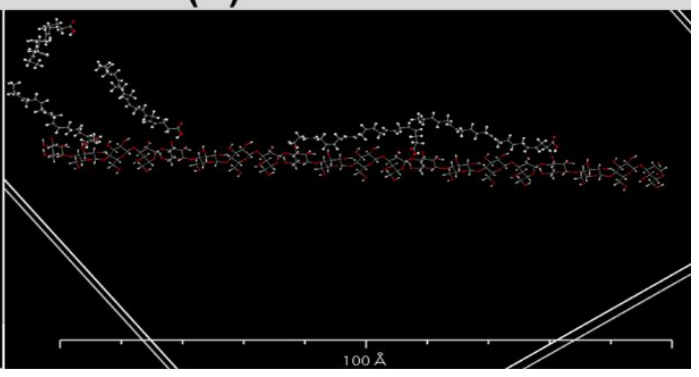

Supporting Figure S5. Low and high energy configuration (LEC, HEC) obtained through MC simulation for the $\mathrm{BG}-\mathrm{H}_{2} \mathrm{O}$ system $(\mathrm{A}, \mathrm{B})$ and the dry BG-DHA system $(\mathrm{C}, \mathrm{D})$.

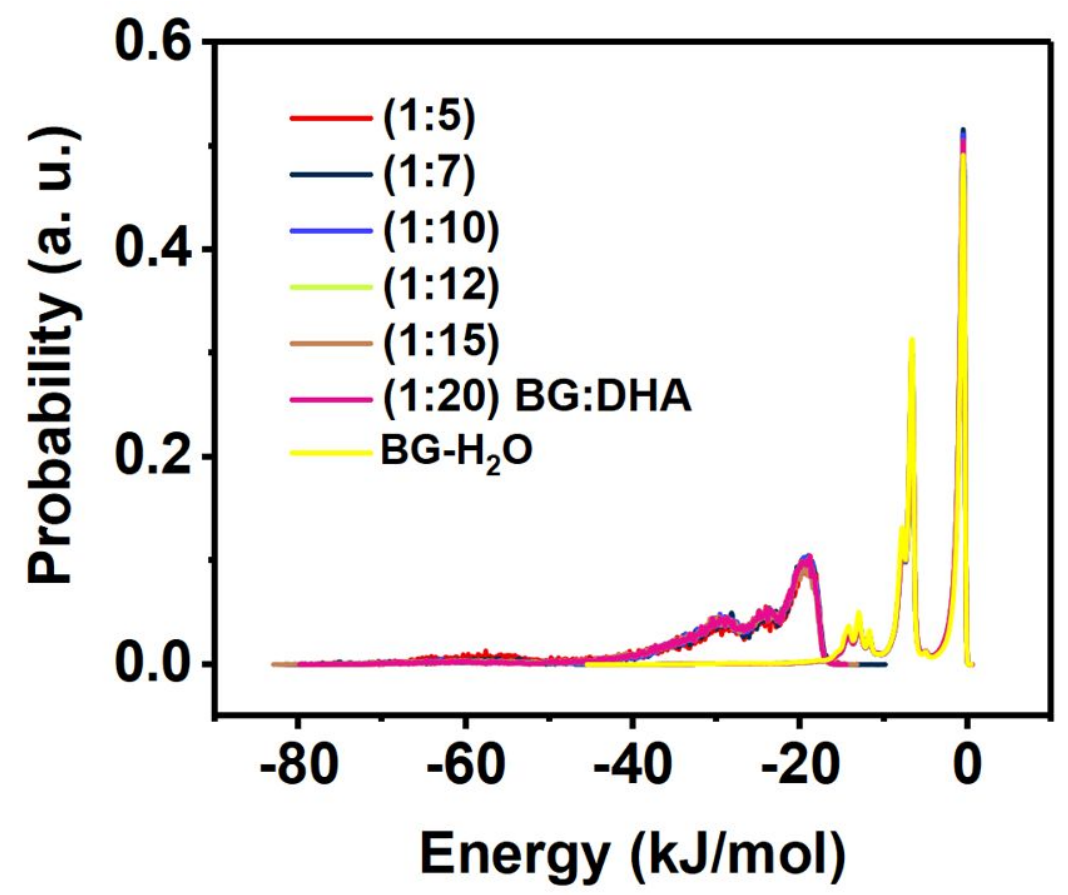

Supporting Figure S6. The energy distribution probability for interaction between BG, DHA, and $\mathrm{H}_{2} \mathrm{O}$ molecules. The colored legends show the corresponding systems for $\mathrm{BG}-\mathrm{H}_{2} \mathrm{O}$, BG- 
5DHA(aq), BG-7DHA(aq), BG-10DHA(aq), BG-12DHA(aq), BG-15DHA(aq), and BG20DHA(aq).
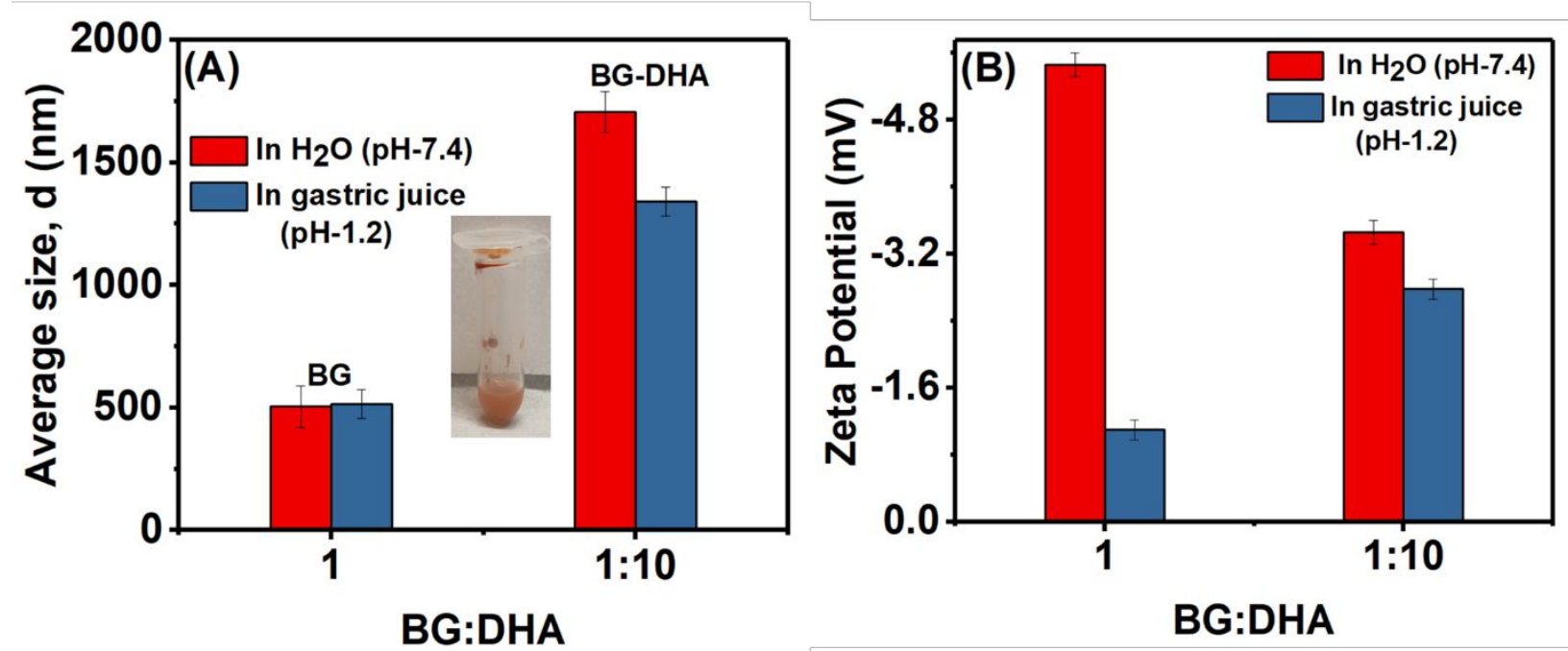

Supporting Figure S7. Comparison plot of DLS size (A) and zeta potential measurement (B) of BG and BG:DHA system in gastric juice of $\mathrm{pH}$ 1.0-1.2 and neutral aqueous solution of $\mathrm{pH}$ 7.4. 

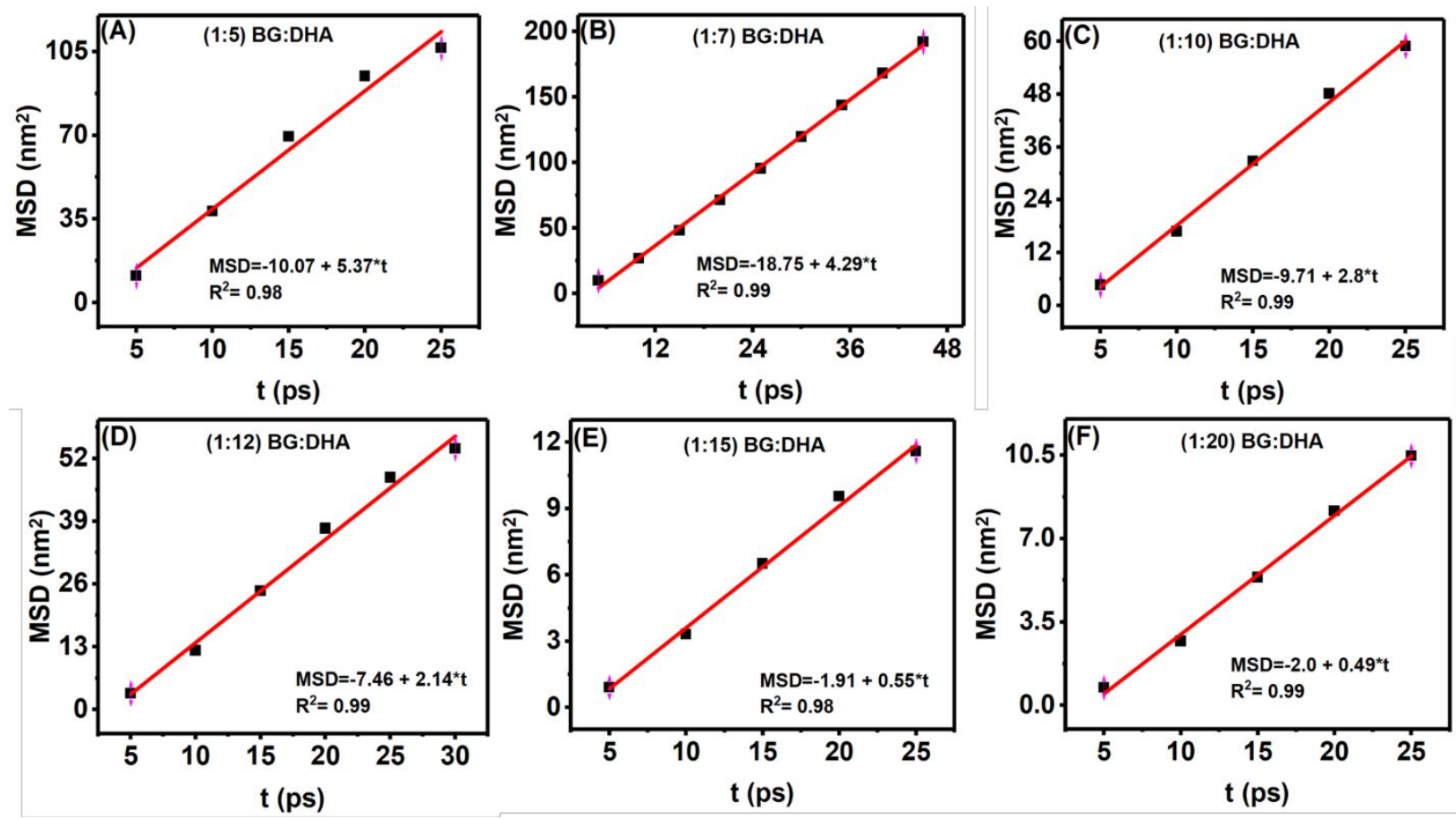

Supporting Figure S8. Mean square displacement (MSD) obtained for six systems through MD simulation at a constant temperature $(310 \mathrm{~K})$ and volume (NPT).
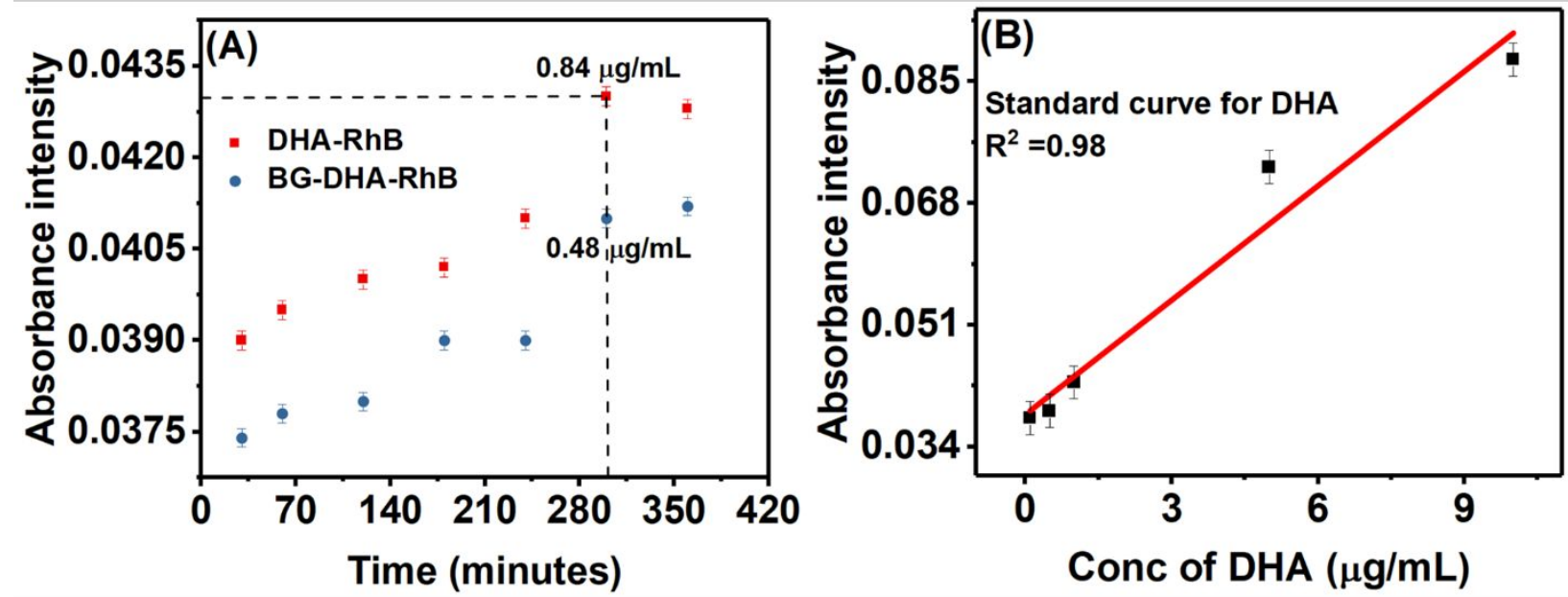

Supporting Figure S9. Study of the in vitro intestinal permeation of the DHA-RhB and BG-DHARhB formulations. Figure A shows the endpoint absorbance value of the collected samples 
diffused through the intestinal tract $(n=3)$ and obtained at seven different time periods of 30 mins, $1,2,3,4,5$, and $6 \mathrm{hrs}$, respectively. The concentration of the maximum DHA diffused through the intestine are included in the figure. Figure B shows the standard absorbance vs concentration of DHA plot obtained for DHA-RhB system. This plot was used for determining the concentration of the DHA that were penetrated through intestine during permeation study.

Supporting Table S1. Typical IR absorption bands $\left(\mathrm{cm}^{-1}\right)$ obtained for different functional groups for both simulated and experimental BG and DHA molecules and for simulated BG-DHA system.

\begin{tabular}{|c|c|c|c|c|c|c|c|c|c|}
\hline \multicolumn{2}{|c|}{$\begin{array}{c}\text { Simulated IR } \\
\text { for BG }\end{array}$} & \multicolumn{2}{|c|}{$\begin{array}{l}\text { Experimental } \\
\text { IR for BG }\end{array}$} & \multicolumn{2}{|c|}{$\begin{array}{c}\text { Simulated IR } \\
\text { for DHA }\end{array}$} & \multicolumn{2}{|c|}{$\begin{array}{l}\text { Experimental } \\
\text { IR for DHA }\end{array}$} & \multicolumn{2}{|c|}{$\begin{array}{c}\text { IR for BG-DHA } \\
\text { system }\end{array}$} \\
\hline $\mathrm{FG}$ & $\mathrm{cm}^{-1}$ & $\mathrm{FG}$ & $\mathrm{cm}^{-1}$ & $\mathrm{FG}$ & $\mathrm{cm}^{-1}$ & $\mathrm{FG}$ & $\mathrm{cm}^{-1}$ & $\mathrm{FG}$ & $\mathrm{cm}^{-1}$ \\
\hline $\begin{array}{c}\text { Strong } \\
\text { O-H } \\
\text { stretching }\end{array}$ & $\begin{array}{l}3500- \\
3000\end{array}$ & $\begin{array}{c}\text { Strong } \\
\text { O-H } \\
\text { stretching }\end{array}$ & $\begin{array}{l}3500- \\
3000\end{array}$ & $\begin{array}{c}\text { Weak O- } \\
\mathrm{H} \\
\text { stretching }\end{array}$ & $\begin{array}{l}3500- \\
3100\end{array}$ & & & $\begin{array}{c}\text { Strong C- } \\
\mathrm{H} \\
\text { stretching }\end{array}$ & $\begin{array}{l}2950- \\
2800\end{array}$ \\
\hline $\begin{array}{c}\text { Weak C- } \\
\text { H } \\
\text { stretching }\end{array}$ & $\begin{array}{l}3000- \\
2850\end{array}$ & $\begin{array}{c}\text { Weak C- } \\
\text { H } \\
\text { stretching }\end{array}$ & $\begin{array}{l}3000- \\
2800\end{array}$ & $\begin{array}{c}\text { Strong } \\
=\mathrm{C}-\mathrm{H} \\
\text { stretching }\end{array}$ & $\begin{array}{l}3100- \\
3006\end{array}$ & $\begin{array}{c}\text { Weak } \\
=\mathrm{C}-\mathrm{H} \\
\text { stretching }\end{array}$ & $\begin{array}{l}3012- \\
3006\end{array}$ & $\begin{array}{c}\text { Strong } \\
\mathrm{O}=\mathrm{C}=\mathrm{O} \\
\text { stretching }\end{array}$ & 2396 \\
\hline & & $\begin{array}{c}\text { Weak } \\
\mathrm{H}_{2} \mathrm{O} \\
\text { stretching }\end{array}$ & 1638 & $\begin{array}{c}\text { Weak C- } \\
\mathrm{H} \\
\text { stretching }\end{array}$ & $\begin{array}{l}3000- \\
2850\end{array}$ & $\begin{array}{c}\text { Strong C- } \\
\text { H } \\
\text { stretching }\end{array}$ & $\begin{array}{l}3000- \\
2850\end{array}$ & & \\
\hline $\begin{array}{c}\text { Strong C- } \\
\text { H } \\
\text { bending } \\
\left(\mathrm{CH}_{2}\right)\end{array}$ & $\begin{array}{l}1500- \\
1465\end{array}$ & & & $\begin{array}{c}\text { Strong } \\
\mathrm{C}=\mathrm{O} \\
\text { stretching }\end{array}$ & 1766 & $\begin{array}{c}\text { Strong } \\
\mathrm{C}=\mathrm{O} \\
\text { stretching }\end{array}$ & $\begin{array}{l}1750- \\
1770\end{array}$ & $\begin{array}{c}\text { Weak } \\
\mathrm{C}=\mathrm{C} \\
\text { stretching }\end{array}$ & $\begin{array}{l}1670- \\
1600\end{array}$ \\
\hline $\begin{array}{c}\text { Weak C- } \\
\text { H } \\
\text { bending } \\
\left(\mathrm{CH}_{3}\right)\end{array}$ & $\begin{array}{l}1450- \\
1300\end{array}$ & $\begin{array}{c}\text { Weak C- } \\
\text { H } \\
\text { bending }\end{array}$ & $\begin{array}{l}1465- \\
1300\end{array}$ & $\begin{array}{c}\text { Strong C- } \\
\qquad \mathrm{H} \\
\text { bending } \\
\quad\left(\mathrm{CH}_{2}\right. \\
\left.\mathrm{CH}_{3}\right)\end{array}$ & $\begin{array}{l}1465- \\
1300\end{array}$ & $\begin{array}{c}\text { Weak C- } \\
\qquad \mathrm{H} \\
\text { bending } \\
\left(\mathrm{CH}_{2} \text {, }\right. \\
\left.\mathrm{CH}_{3}\right)\end{array}$ & $\begin{array}{l}1465- \\
1300\end{array}$ & $\begin{array}{c}\text { Strong C- } \\
\mathrm{H} \\
\text { bending } \\
\left(\mathrm{CH}_{2}\right)\end{array}$ & 1470 \\
\hline
\end{tabular}




\begin{tabular}{|c|c|c|c|c|c|c|c|c|c|}
\hline $\begin{array}{c}\text { Strong C- } \\
\text { OH }\end{array}$ & $\begin{array}{l}1200- \\
1000\end{array}$ & $\begin{array}{c}\text { Strong C- } \\
\mathrm{OH}\end{array}$ & $\begin{array}{l}1200- \\
1000\end{array}$ & $\begin{array}{c}\text { Strong C- } \\
\text { O } \\
\text { stretching }\end{array}$ & $\begin{array}{l}1200- \\
1000\end{array}$ & $\begin{array}{l}\text { Strong C- } \\
\text { O } \\
\text { stretching }\end{array}$ & $\begin{array}{l}1200- \\
1000\end{array}$ & $\begin{array}{l}\text { Weak C- } \\
\text { O } \\
\text { stretching }\end{array}$ & $\begin{array}{l}1200- \\
1000\end{array}$ \\
\hline $\begin{array}{l}\text { Weak } \beta \text { - } \\
\text { glucan } \\
\text { linkage }\end{array}$ & 885 & $\begin{array}{c}\beta \text {-glucan } \\
\text { linkage }\end{array}$ & 894 & $\begin{array}{c}\text { Strong } \\
\mathrm{C}=\mathrm{C}-\mathrm{H} \\
\text { bending }\end{array}$ & 900 & $\begin{array}{l}\mathrm{C}=\mathrm{C}-\mathrm{H} \\
\text { bending }\end{array}$ & 900 & $\begin{array}{c}\text { Strong } \beta \text { - } \\
\text { glucan } \\
\text { linkage }\end{array}$ & 870 \\
\hline
\end{tabular}

Supporting Table S2: Different BG-DHA-water mixtures used in bulk computational simulation where 1000 water molecules were used.

\begin{tabular}{|c|c|c|c|c|}
\hline $\begin{array}{c}\text { No of } \\
\text { experiments }\end{array}$ & $\begin{array}{c}\text { No of } \\
\text { repeating } \\
\text { units of } \\
\text { Beta } \\
\text { glucan }\end{array}$ & $\begin{array}{c}\text { No } \\
\text { of } \\
\text { DHA }\end{array}$ & $\begin{array}{l}\text { No of } \\
\text { water }\end{array}$ & System \\
\hline 1 & 5 & & 1000 & $\mathrm{BG}-\mathrm{H}_{2} \mathrm{O}$ \\
\hline 2 & 5 & 5 & & BG-DHA \\
\hline 3 & 5 & 5 & 1000 & BG-5DHA(aq)/(1:5) BG:DHA \\
\hline 4 & 5 & 7 & 1000 & BG-7DHA(aq)/(1:7) BG:DHA \\
\hline 5 & 5 & 10 & 1000 & BG-10DHA(aq)/(1:10) BG:DHA \\
\hline 6 & 5 & 12 & 1000 & BG-12DHA(aq)/(1:12) BG:DHA \\
\hline 7 & 5 & 15 & 1000 & BG-15DHA(aq)/(1:15) BG:DHA \\
\hline 8 & 5 & 20 & 1000 & BG-20DHA(aq)/(1:20) BG:DHA \\
\hline
\end{tabular}


Supporting Table S3: Different BG-DHA-water mixtures used in bulk computational simulation where 50 water molecules were used.

\begin{tabular}{|c|c|c|c|c|}
\hline No of & $\begin{array}{c}\text { No of } \\
\text { experiments }\end{array}$ & No of DHA & No of water & System \\
units of & & & & \\
\hline $\mathbf{1}$ & Beta glucan & & & \\
\hline $\mathbf{2}$ & 5 & & 50 & BG_H2O(50) \\
\hline $\mathbf{3}$ & 5 & 5 & & BG_DHA \\
\hline $\mathbf{4}$ & 5 & 5 & 50 & BG_DHA(5) \\
\hline $\mathbf{5}$ & 5 & 10 & 50 & BG_DHA(10) \\
\hline $\mathbf{6}$ & 5 & 12 & 50 & BG_DHA(12) \\
\hline $\mathbf{7}$ & 5 & 15 & 50 & BG_DHA(15) \\
\hline $\mathbf{8}$ & 5 & 20 & 50 & BG_DHA(20) \\
\hline & 5 & 7 & 50 & BG_DHA(7) \\
\hline
\end{tabular}

Supporting Table S4. Energy (in $\mathrm{kJ} / \mathrm{mol}$ ) values computed through $\mathrm{MC}$ simulation for eight different systems having $\mathrm{BG}$ (three repeating units), DHA and $\mathrm{H}_{2} \mathrm{O}$ (up to 50 molecules). The energies for the most stable (low adsorption energy) and the least energy (high adsorption energy) configurations are shown for each system.

\begin{tabular}{|c|c|c|c|c|c|c|}
\hline $\begin{array}{c}\text { BG-DHA- } \\
\mathrm{H}_{2} \mathrm{O} \\
\text { systems }\end{array}$ & $\begin{array}{c}\text { Total } \\
\text { Energy }\end{array}$ & $\begin{array}{c}\text { Adsorptio } \\
\text { n Energy } \\
(\mathbf{R}+\mathrm{D})\end{array}$ & $\begin{array}{c}\text { Rigid Adsorption } \\
\text { Energy }\end{array}$ & $\begin{array}{c}\text { Deformation } \\
\text { Energy }\end{array}$ & $\mathrm{H}_{2} \mathrm{O}: \mathrm{dEad} / \mathrm{dNi}$ & DHA:dEad/dNi \\
& & & & $(\mathrm{D})$ & & \\
\end{tabular}




\begin{tabular}{|c|c|c|c|c|c|c|}
\hline $\begin{array}{c}\text { BG- } \\
\mathrm{H}_{2} \mathrm{O}(50)- \\
\text { LEC }\end{array}$ & -632.95 & -642.57 & -661.94 & 19.37 & -0.23 & - \\
\hline $\begin{array}{c}\text { BG- } \\
\mathrm{H}_{2} \mathrm{O}(50)- \\
\text { HEC }\end{array}$ & -541.77 & -551.39 & -568.11 & 16.72 & -0.2 & - \\
\hline $\begin{array}{l}\text { BG-DHA- } \\
\text { LEC }\end{array}$ & -407.6 & -277.83 & -211.14 & -66.7 & - & -36.4 \\
\hline $\begin{array}{l}\text { BG-DHA- } \\
\text { HEC }\end{array}$ & -307.63 & -177.87 & -92.87 & -84.99 & - & -17.68 \\
\hline $\begin{array}{l}\text { BG- } \\
\text { 5DHA(aq)- } \\
\text { LEC }\end{array}$ & -958.05 & -837.86 & -779.81 & -58.05 & -0.23 & -42.88 \\
\hline $\begin{array}{c}\text { BG- } \\
\text { 5DHA(aq)- } \\
\text { HEC }\end{array}$ & -858.87 & -738.73 & -679.02 & -59.71 & -0.18 & -32.04 \\
\hline $\begin{array}{l}\text { BG-7DHA- } \\
\text { LEC }\end{array}$ & -902.45 & -730.41 & -640.08 & -90.33 & -0.16 & -37.43 \\
\hline $\begin{array}{l}\text { BG-7DHA- } \\
\text { HEC }\end{array}$ & -802.49 & -630.45 & -530.5 & -99.95 & -0.18 & -24.75 \\
\hline $\begin{array}{l}\text { BG- } \\
\text { 10DHA- } \\
\text { LEC }\end{array}$ & -1281.47 & -1031.57 & -896.89 & -134.68 & -0.29 & -29.79 \\
\hline $\begin{array}{l}\text { BG- } \\
\text { 10DHA- } \\
\text { HEC }\end{array}$ & -1182.82 & -932.92 & -798.72 & -134.2 & -0.24 & -26.38 \\
\hline $\begin{array}{l}\text { BG- } \\
\text { 12DHA- } \\
\text { LEC }\end{array}$ & -1333.19 & -1031.38 & -857.78 & -173.6 & -0.22 & -34.6 \\
\hline
\end{tabular}




\begin{tabular}{|c|c|c|c|c|c|c|}
\hline $\begin{array}{l}\text { BG- } \\
\text { 12DHA- } \\
\text { HEC }\end{array}$ & -1234.38 & -932.57 & -752.19 & -180.38 & -0.21 & -25.95 \\
\hline $\begin{array}{l}\text { BG- } \\
\text { 15DHA- } \\
\text { LEC }\end{array}$ & -1497.64 & -1117.98 & -901.29 & -216.68 & -0.17 & -18.09 \\
\hline $\begin{array}{l}\text { BG- } \\
\text { 15DHA- } \\
\text { HEC }\end{array}$ & -1397.88 & -1018.22 & -793.82 & -224.39 & -0.18 & -17.99 \\
\hline $\begin{array}{l}\text { BG- } \\
\text { 20DHA- } \\
\text { LEC }\end{array}$ & -1871.94 & -1362.51 & -1065.96 & -296.55 & -0.19 & -18.42 \\
\hline $\begin{array}{l}\text { BG- } \\
\text { 20DHA- } \\
\text { HEC }\end{array}$ & -1772.41 & -1262.98 & -959.43 & -303.56 & -0.18 & -17.86 \\
\hline \multicolumn{7}{|c|}{$\begin{array}{l}\mathrm{dEad} / \mathrm{dNi} \text { : is the desorption energy required to release an adsorbed species from the substrate. } \\
\text { Deformation energy: is the energy being released after adsorption. } \\
\text { Rigid adsorption energy is the energy for the adsorption of an unoptimized molecule on the substrate. } \\
\text { Adsorption energy: is the energy necessary for the adsorption of a flexible optimized structure on the substrate molecule } \\
\text { Total energy: adsorption energy- (total energy of adsorbate } \times \text { total number of adsorbate molecules) } \\
\text { LEC and HEC: low energy and high energy configurations. }\end{array}$} \\
\hline
\end{tabular}

Supporting Table S5. Binding energy calculation for eight systems. The BE calculation was carried out based on the energy values obtained from geometry optimization process.

\begin{tabular}{|c|c|c|c|c|c|}
\hline System & $\begin{array}{c}\text { Total energy } \\
\text { of the } \\
\text { system } \\
(\mathrm{kJ} / \mathrm{mol})\end{array}$ & $\begin{array}{c}\text { Energy of } \\
\text { BG }(\mathrm{kJ} / \mathrm{mol})\end{array}$ & $\begin{array}{c}\text { Energy of } \\
\mathrm{H}_{2} \mathrm{O}(\mathrm{kJ} / \mathrm{mol})\end{array}$ & $\begin{array}{c}\text { Energy of } \\
\text { DHA } \\
(\mathrm{kJ} / \mathrm{mol})\end{array}$ & $\begin{array}{c}\text { Binding energy } \\
(\mathrm{kJ} / \mathrm{mol})\end{array}$ \\
\hline BG & & -188.16 & & & \\
\hline DHA & & & & -45.14 & \\
\hline $\mathrm{H}_{2} \mathrm{O}$ & & & -4.06 & & \\
\hline BG-DHA & -437.14 & -188.16 & & $-45.14 \times 5$ & -23.28 \\
\hline BG- $\mathrm{H}_{2} \mathrm{O}$ & -4255.62 & -188.16 & $-4.06 \times 1000$ & & -7.46 \\
\hline
\end{tabular}




\begin{tabular}{|l|l|l|l|l|l|}
\hline BG-5DHA & -4496.16 & -188.16 & $-4.06 \times 1000$ & $-45.14 \times 5$ & -22.3 \\
\hline BG-7DHA & -4586.31 & -188.16 & $-4.06 \times 1000$ & $-45.14 \times 7$ & -22.21 \\
\hline BG-10DHA & -4724.42 & -188.16 & $-4.06 \times 1000$ & $-45.14 \times 10$ & -24.86 \\
\hline BG-12DHA & -4818.68 & -188.16 & $-4.06 \times 1000$ & $-45.14 \times 12$ & -28.84 \\
\hline BG-15DHA & -4952.15 & -188.16 & $-4.06 \times 1000$ & $-45.14 \times 15$ & -26.89 \\
\hline BG-20DHA & -5178.01 & -188.16 & $-4.06 \times 1000$ & $-45.14 \times 20$ & -27.05 \\
\hline
\end{tabular}

